\title{
Anode plasma dynamics in the self-magnetic-pinch diode
}

\author{
Nichelle Bruner and Dale R. Welch \\ Voss Scientific, LLC, Albuquerque, New Mexico 87108, USA \\ Kelly D. Hahn and Bryan V. Oliver \\ Sandia National Laboratories, Albuquerque, New Mexico 81185, USA
}

(Received 23 November 2010; published 2 February 2011)

\begin{abstract}
The self-magnetic-pinch diode is being developed as an intense electron beam source for pulsed-powerdriven x-ray radiography. In high-power operation, the beam electrons desorb contaminants from the anode surface from which positive ions are drawn to the cathode. The counterstreaming electrons and ions establish an equilibrium current. It has long been recognized, however, that expanding electrode plasmas can disrupt this equilibrium and cause rapid reduction of the diode impedance and the radiation pulse. Recently developed numerical techniques, which enable simultaneous modeling of particle currents with $10^{13} \mathrm{~cm}^{-3}$ densities to plasmas of near solid density, are applied to a model of the self-magnetic-pinch diode which includes the formation and evolution of anode surface plasmas. Two mechanisms are shown to cause rapid impedance loss, anode plasma expansion into the anode-cathode (A-K) gap, and increased ion space-charge near the cathode surface. The former mechanism dominates for shorter A-K gaps, while the latter dominates for longer gaps. Model results qualitatively reproduce the time-dependent impedances measured for this diode.
\end{abstract}

DOI: 10.1103/PhysRevSTAB.14.024401

\section{INTRODUCTION}

The self-magnetic-pinch (SMP) diode [1-4] is being developed as an intense electron beam source for pulsedpower-driven x-ray radiography. The diode is composed of a thin cylindrical cathode with a hollow, rounded tip, as illustrated in Fig. 1. As the electron beam emitted from the cathode traverses the gap to the planar anode, it pinches due to its self-magnetic force, which is balanced by its repulsive self-electric force. The anode is composed of a thin Al foil placed on top of a high-atomic-number material which efficiently converts the beam electrons into $\mathrm{x}$ rays via bremsstrahlung.

During operation, the electron beam deposits sufficient energy to quickly heat the anode, desorbing hydrocarbon contaminants and creating an anode surface plasma. A space-charge-limited (SCL) ion current is drawn from this plasma which counterstreams with the electron beam toward the cathode. The ion space-charge partially neutralizes the beam, thereby enabling an increased pinch. A new equilibrium is established with these counterstreaming currents, resulting in a larger diode current than in monopolar flow [5]. The diode operates as a current-limited device with the equilibrium current determined by a critical value of $[2,6]$

$$
I_{\text {crit }}=8.5 \alpha \frac{r_{C}}{g}\left(\gamma^{2}-1\right)^{1 / 2} \quad[\mathrm{kA}],
$$

where $\gamma=1+e V / m_{e} c^{2}$ is the relativistic factor, $r_{C}$ is the cathode radius, $g$ is the anode-cathode (A-K) gap width, and $\alpha$ is a scale factor used to account for the current increase due to ion space charge. Estimates of $\alpha$ range
PACS numbers: 41.85.Ja, 52.25.Fi, 52.65.Rr, 52.65.Ww

from 1.6 to 2.8, depending on the diode geometry and the beam and ion space-charge profiles.

It has been observed, however, that the SMP diode current increases beyond predictions for the critical current, resulting in a decreasing diode impedance $[4,7,8]$. The proposed mechanism for the impedance reduction is electrode plasma expansion into the A-K gap [3,9].

An example of the falling impedance of the SMP diode is shown in data from Sandia National Laboratories' Radiographic Integrated Test Stand (RITS-6) pulsedpower accelerator [10]. With a $40-\Omega$ magnetically insulated transmission line, the diode nominally produces $150 \mathrm{kA}$ at 7.5 MV. The time-dependent impedances for

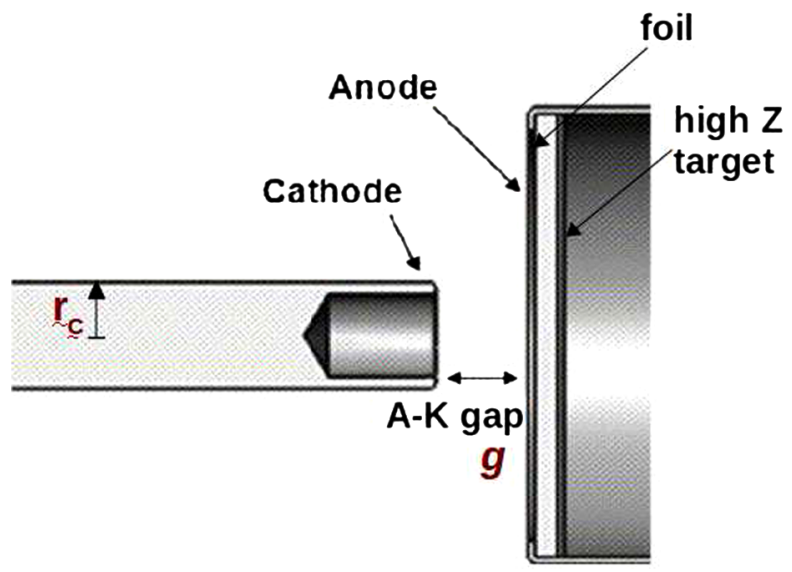

FIG. 1. Cross-sectional diagram of the SMP diode. The diode geometries considered here are distinguished by their ratio of anode-cathode (A-K) gap to cathode radius $\left(g / r_{C}\right)$. 
the diode for different aspect ratios of A-K gap width-tocathode radius $\left(g / r_{C}\right)$ are shown in Fig. 2. From Eq. (1) the diode impedance is expected to increase with $g / r_{C}$. At relatively early times ( $<35 \mathrm{~ns}$ ), this trend is observed in the measured impedances shown in Fig. 2. At later times, the smallest and largest values $g / r_{C}(1.63$ and 2.08) show a rapid fall in impedance, dropping below $10 \Omega$ before the end of the pulse. In contrast, the impedances for the intermediate values of $g / r C(1.76$ and 1.92) fall at a much much slower rate. (A stable-impedance history provides for a longer duration radiation pulse.) These dramatic changes in impedance histories occur over a relatively small range of $g / r C$ values. Understanding the physical mechanisms governing this small, stable-impedance parameter window is important to extending the diode operation to higher powers.

A recent publication highlights new simulation techniques for electrode plasmas [11] which are applied here to the SMP diode. A hybrid particle-in-cell (PIC) technique enables efficient, simultaneous modeling of the dense $\left(\lessgtr 10^{18} \mathrm{~cm}^{-3}\right.$ ) electron and ion populations associated with electrode surface plasmas and the lower-density $\left(\sim 10^{13}-10^{15} \mathrm{~cm}^{-3}\right)$ populations occurring in bipolar flow. The dense populations are modeled as inertial Eulerian fluids. As the fluid particles accelerate above a prescribed kinetic energy threshold, they transition into a kinetic-macroparticle treatment. The hybrid algorithms have been incorporated into the implicit electromagnetic PIC code LSP [12]. To improve understanding of the impedance behavior of the SMP diode, detailed simulations are performed using LSP which include the creation and evolution of an anode plasma.

The parameters used in the diode simulations are discussed in Sec. II. Simulation results are discussed in Sec. III with emphasis on the mechanisms which lead to the observed falling diode impedance. Conclusions are summarized in Sec. IV.

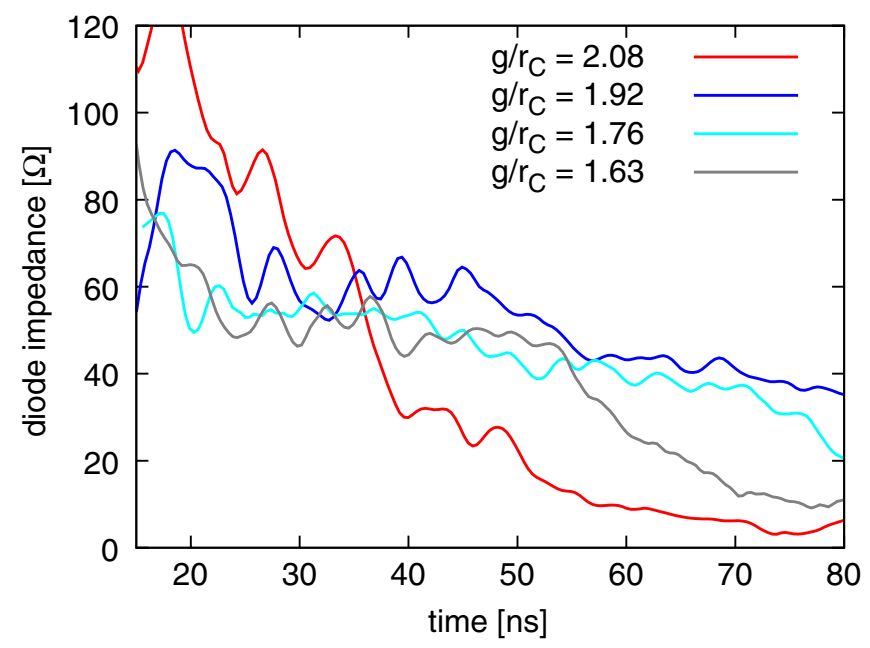

FIG. 2. The SMP diode impedance measured on RITS-6 for four values of $g / r_{C}$.

\section{MODEL PARAMETERS FOR THE SMP DIODE}

The parameters used in simulations of the SMP diode using the hybrid, implicit PIC code LSP are described here. The diode dimensions and peak power are chosen to emulate the experiments recently performed on RITS-6 [4]. The value of $r_{C}$ is fixed while $g / r_{C}$ is varied from 1.12 to 2.08. A voltage pulse with $7.5 \mathrm{MV}$ peak amplitude and an 8-ns rise is used to drive the diode. (The actual pulse rise on RITS-6 is closer to $15 \mathrm{~ns}$, which does not significantly delay anode heating.) The simulations are conducted in $2 \mathrm{D}$ cylindrical coordinates $(r, z)$ with $80-\mu \mathrm{m}$ grid resolution. Figure 3 shows the simulation geometry which is axially truncated from the actual hardware in order to reduce the size of the simulation space and, therefore, the computation time.

SCL electron emission [5,13] is modeled along the cathode in the region outlined in Fig. 3, with an electric field threshold of $150 \mathrm{kV} / \mathrm{cm}$. A region of anode plasma formation is also outlined in Fig. 3, indicating the extent of electron beam heating. Recent spectroscopic measurements on RITS- 6 have observed the formation of an anode plasma prior to the measured radiation pulse, which migrates into the A-K gap during the pulse [14]. A cathode plasma begins to appear during the radiation pulse, well after the anode plasma and with less intensity. For this reason, a cathode plasma is neglected in this model.

The anode plasma is generated using a two-step method which minimizes nonphysical sheath formation at the injection plane. First, a neutral gas is desorbed from the anode surface and allowed to migrate some prescribed number of grid cells away from the conductor (two to three cells are used here). Then the neutral gas fragments into a plasma. Two mechanisms are simultaneously used to desorb neutral particles from the anode surface. One is stimulated desorption in which neutral particles are liberated from the anode surface by individual high-energy

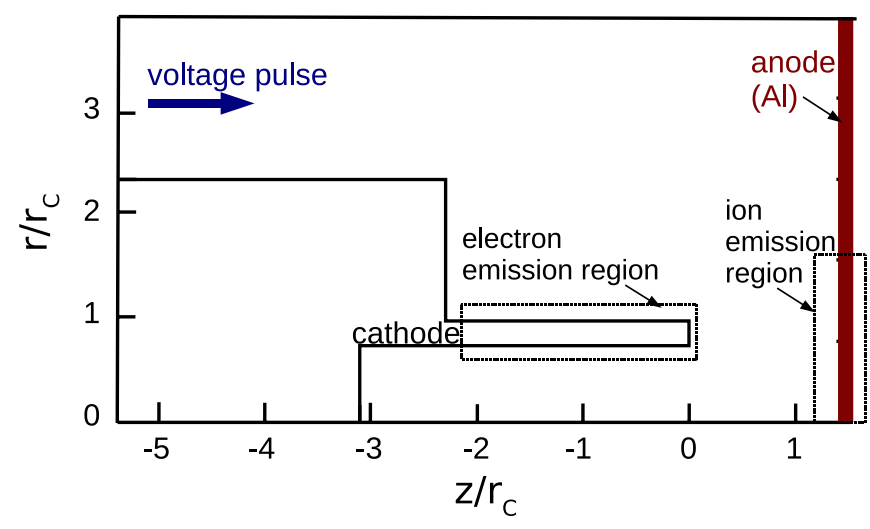

FIG. 3. The simulation geometry of the SMP diode. The forward-going voltage wave is injected at the upper left. The region of electron SCL emission is outlined on the cathode. Desorption of the anode plasma occurs in the region outlined on the anode. 
electrons. This is also referred to as electron-induced desorption. The second is thermal desorption, in which neutral particles desorb due to an increase in the anode material's surface temperature.

In the stimulated desorption model, neutral desorption is initiated by electron impact at a rate of $\Delta n_{n}=n_{e} \sigma n_{\mathrm{ML}}$, where $n_{e}$ is incident electron density, $\sigma$ is the stimulated desorption cross section, and $n_{\mathrm{ML}}$ is the number of monolayers (ML) of surface contaminants. The value of $\sigma$ used here is $1.25 \times 10^{-19} \mathrm{~cm}^{2}$, consistent with Refs. [15-17]. A monolayer is assumed to have $10^{15} \mathrm{~cm}^{-2}$ areal density. Experiments have shown that under vacuum, an electrode surface can accrue over 45 monolayers of contaminants in 24 hours after cleaning [18]. Since cleaning is not performed on RITS-6, 40 monolayers are assumed here.

In the thermal desorption model, ion emission is a function of the surface temperature. The temperature increase $(\Delta T)$ is calculated from the energy deposited per length $(d E / d x)$ by the impacting electrons with charge $q$ via $\Delta T=d E / d x q /\left(e \rho N_{\mathrm{av}} C_{p} \cos \theta\right)$, where $\rho$ is the material density, $N_{\mathrm{av}}$ is Avogadro's number, and $\theta$ is the electron angle of incidence. The ion emission rate is [19]

$$
\frac{d n_{n}}{d t}=\nu_{\mathrm{th}} n_{\mathrm{ML}} e^{-Q_{a} /\left[R T_{a}(t)\right]},
$$

where $\nu_{\mathrm{th}} \sim 10^{13} \mathrm{~s}^{-1}, Q_{a}$ is the binding energy $\left(Q_{a} \sim\right.$ $23 \mathrm{kcal} / \mathrm{mol}$ ), and $R=1.9858 \times 10^{-3} \mathrm{kcal} / \mathrm{mol} / \mathrm{K}$.

The anode plasma is expected to be primarily composed of water and hydrocarbon surface contaminants and ablated target material [20,21]. The surface contaminants serve as a source for protons which are commonly found in experiments involving intense electron beam impact [21-23]. Simulations modeling a hydrogen anode plasma as described above show depletion of the plasma density as protons are drawn into bipolar flow, and plasma expansion does not occur. This is consistent with the initial desorption rates $(<1 \mathrm{ML} / \mathrm{ns})$ and the proton current predicted from the ratio of the ion-to-electron SCL bipolar current densities in Ref. [5],

$$
\frac{J_{i}}{J_{e}}=\frac{m_{e}}{m_{i}} \frac{\sqrt{\gamma_{e}^{2}-1}}{\sqrt{\gamma_{i}^{2}-1}}
$$

At 7.5 MV, $J_{i} / J_{e}=0.07$ for protons which approaches $1 \mathrm{ML} / \mathrm{ns}$, depending on the beam radius. Since the desorption rates are not considered functions of the ion mass, the smaller currents associated with heavier (singly ionized) ion species are less likely to deplete the desorbed plasma. Spectroscopy results from RITS-6 for the SMP and paraxial diodes show dominant populations of carbon and aluminum ions in the $10^{14}-10^{15} \mathrm{~cm}^{-3}$ range for an aluminum covered anode target [14]. Therefore, the anode plasmas studied here are composed of singly ionized carbon or aluminum.

\section{MECHANISMS FOR DIODE-IMPEDANCE LOSS}

The impedance lifetime of the SMP diode can be viewed as having three stages. The first is monopolar flow, when (after cathode plasma formation) electrons are emitted from the cathode but have not sufficiently heated the anode to desorb surface particles. The second stage is the bipolar flow described in Sec. I. In the third stage, the current rises above bipolar flow at a rate apparently determined by the A-K gap width, as indicated in Fig. 2.

In simulations without an electrode plasma, only the first two stages of diode impedance occur. The diode current evolves only to bipolar flow, as described by Eq. (1). An example of the time-dependent impedance for this case is shown in Fig. 4(b) (in green). This simulation uses the Fig. 3 geometry with $g / r_{C}=1.12$. $\mathrm{Al}^{+}$ions are emitted from the anode after roughly $7 \mathrm{~ns}$ when the surface temperature increases by $400 \mathrm{~K}$. After ion emission, the impedance remains constant. The scale factor $\alpha$ used in Eq. (1) is determined to be 2.1 , but increases to 2.2 for the larger $g / r_{C}$ considered.

When an anode plasma is included in the diode model, all three stages of the impedance lifetime are observed. The
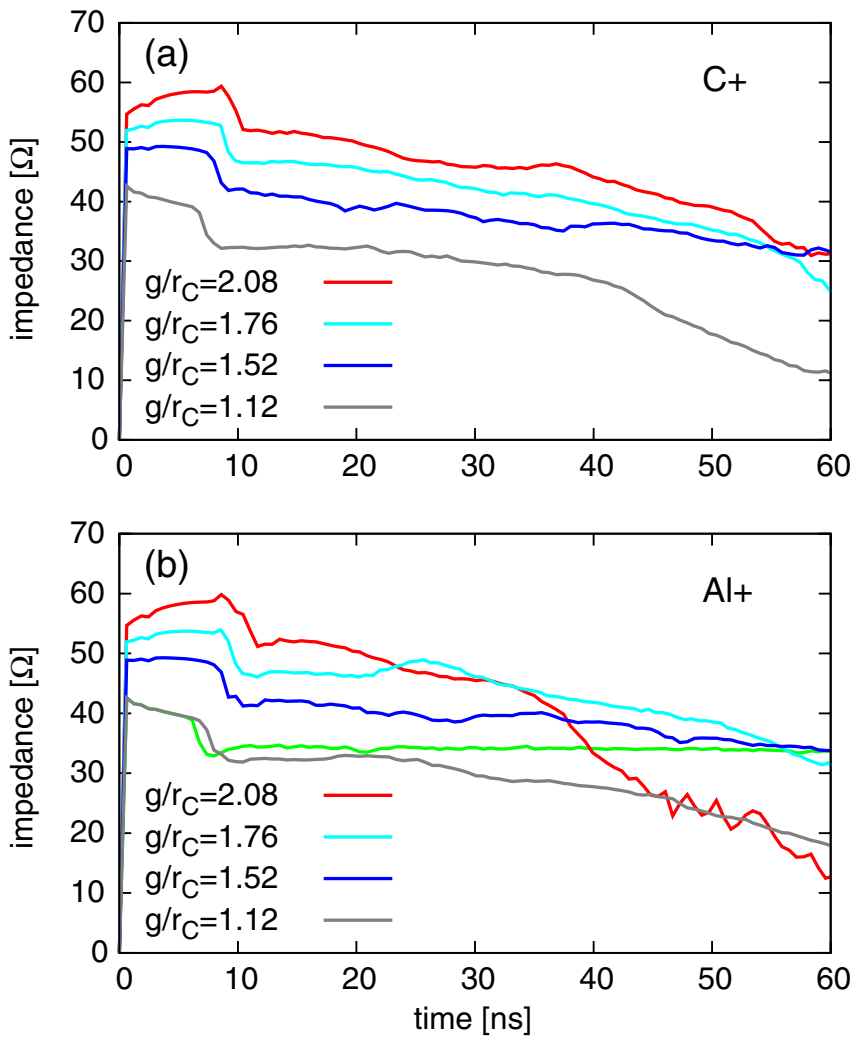

FIG. 4. The simulated time-dependent impedance of the SMP diode for single-species (a) $\mathrm{C}^{+}$and (b) $\mathrm{Al}^{+}$anode plasmas. Results for four values of the diode aspect ratio are shown. The diode impedance shown in green in (b) is from the case of $\mathrm{Al}^{+}$ ion emission without an anode plasma at $g / r_{C}=1.12$. 
time-dependent diode impedances for values of $g / r_{C}$ from 1.12 to 2.08 are shown in Fig. 4(a) for the case of a carbon $\left(\mathrm{C}^{+}\right)$plasma. The monopolar flow exists for the first 7 to 9 ns of the pulse (during the pulse rise), after which the impedances drop to bipolar equilibrium values consistent with Eq. (1). The impedance drop after this stage depends on $g / r_{C}$, with the most rapid drop for $g / r_{C}=1.12$. The diode impedances for the aluminum ( $\left.\mathrm{Al}^{+}\right)$plasma, in Fig. 4 (b), show the most rapid drop for $g / r_{C}=2.08$. While both plasma species exhibit the highest impedance stability for the midrange values of $g / r_{C}$, there is a charge-to-mass ratio $(\mathrm{q} / \mathrm{m})$ dependence to the rates of decline for the larger and smaller values. While the trends observed in Fig. 2 are qualitatively reproduced in Fig. 4, the experimental ion species fraction and distribution of $q / \mathrm{m}$ are not well known.

Simulations show that the impedance decline at the low end of the $g / r_{C}$ range is governed by different dynamics than at the high end. The impedance lifetime for smaller values of $g / r_{C}$ is dominated by plasma expansion into the gap, where a higher $q / m$ for the plasma ions leads to faster expansion. The dense plasma advects the anode potential, effectively reducing the gap width and lowering the diode impedance.

The impedance stability for larger values of $g / r_{C}$ is affected by the dynamics of the ions drawn from the anode plasma. As the anode plasma expands into the gap, the angle the plasma front surface makes with respect to the anode may increase. Ions are liberated perpendicular to the plasma front, and may, therefore, have sufficient transverse momenta to exceed $r_{C}$ before striking the cathode. These ions create greater space charge along the cathode's outer diameter and the electron current increases in response to this space charge. Both mechanisms for impedance loss are discussed in more detail below.

\section{A. Impedance loss for small $g / r_{C}$}

The electron beam desorbs contaminants from the anode which ionize creating a surface plasma. In the hybrid simulations, the plasma ions thus created have low kinetic energy $(1 \mathrm{eV})$ and are treated as a fluid. Ions accelerated

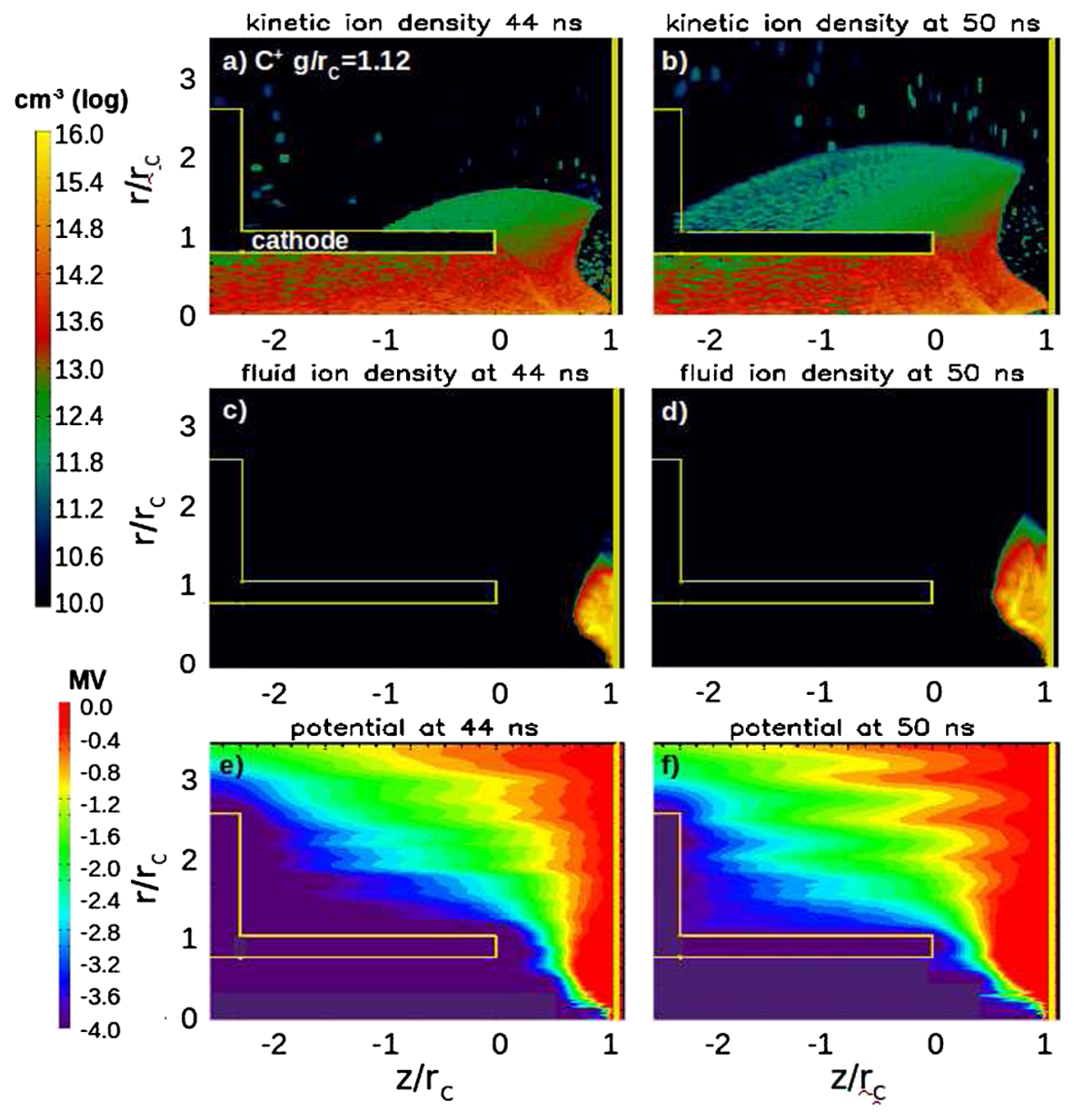

FIG. 5. Snapshots of ion density and electric potential in the SMP diode for a $\mathrm{C}^{+}$anode plasma. The kinetic ( $\left.\geq 200 \mathrm{keV}\right)$ ion density is shown in (a) at $44 \mathrm{~ns}$ and (b) at $50 \mathrm{~ns}$. The plasma density, approximated by fluid ions, is shown in (c) at $44 \mathrm{~ns}$ and (d) at $50 \mathrm{~ns}$. The potential is shown in (e) at $44 \mathrm{~ns}$ and (f) at $50 \mathrm{~ns}$. The density contours are plotted on a $\log _{10}$ scale from $10^{10}-10^{16} \mathrm{~cm}^{-3}$. The potential is plotted on a linear scale from 0 to $-4 \mathrm{MV}$. 
above $200 \mathrm{keV}$ are transitioned into a kinetic particle description. The low-energy ions and associated electrons (treated as a fluid) expand into the gap over the course of the driving pulse at a rate related to the ion thermal velocity (a function of $q / \mathrm{m}$ ) and the gap potential. This expanding plasma advects the anode potential, changing the effective A-K gap width and, therefore, the diode impedance.

While this effect is present for all plasma species and values of $g / r_{C}$, it is easily seen in the example of the $\mathrm{C}^{+}$ anode plasma for $g / r_{C}=1.12$. Figure 5 shows $2 \mathrm{D}$ snapshots of the kinetic and fluid ion densities, and the potential integrated from $E_{z}$, for this simulation. The snapshots are at 44 and $50 \mathrm{~ns}$ into the pulse, well into the period of impedance loss, as shown in Fig. 4. The kinetic ions in Figs. 5(a) and 5(b) form the bipolar current, with densities of $10^{13}-10^{14} \mathrm{~cm}^{-3}$. The low-energy, fluid ions in Figs. 5(c) and 5(d) have $\sim 10^{16} \mathrm{~cm}^{-3}$ density and are seen to expand nonuniformly in $2 \mathrm{D}$ at a time-dependent expansion rate. The potentials integrated from $E_{z}$ in Figs. 5(e) and 5(f) show that the plasma is at the anode potential.

The widths of the effective gaps formed by the anode plasmas are estimated using Figs. 5(e) and 5(f) by measuring the distance from the cathode tip to the closest potential maximum. The actual diode impedances in the Fig. 5 snapshots are approximately equal to values calculated using Eq. (1) with the effective gap widths substituted for the original values. The procedure for determining the effective gap is applied to other small-gap simulations to derive a range of diode-impedance versus effective-gap points, which are plotted in Fig. 6. The impedances for monopolar and bipolar flow, before plasma expansion, are

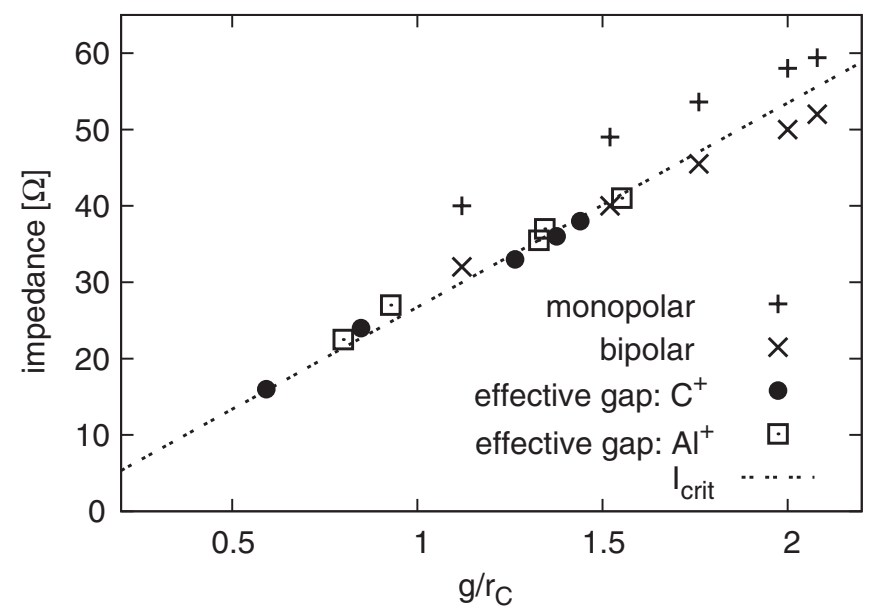

FIG. 6. The SMP diode impedance as a function of $g / r_{C}$. "Monopolar" refers to that portion of the pulse during which only an electron current is present. "Bipolar" refers to counterstreaming electrons and ions. "Effective-gap" measurements are made at 44 and 52 ns into the simulation, after the anode plasma has extended into the A-K gap and changed the location of the potential maximum. The effective gap is shown separately for $\mathrm{C}^{+}$and $\mathrm{Al}^{+}$species anode plasmas. The " $I_{\text {crit }}$ " impedance is calculated from Eq. (1) for $\alpha=2.2$ and $V=7.5 \mathrm{MV}$. also shown. The bipolar values, for both the original and effective gaps, are seen to lie close to the impedance function calculated from Eq. (1) with $\alpha=2.2$ and $V=$ 7.5 MV. The lightest ion species exhibit more rapid gap closure and, for anode plasmas composed of a mixture of ions, the lightest species dictates the gap-closure rate.

The effective-gap points in Fig. 6 are generated from simulations with (original) $g / r_{C} \leq 1.76$. While simulations of larger values of $g / r_{C}$ also exhibit plasma expansion, competing impedance-loss effects are more significant than the decrease in the effective gap. These effects are discussed next.

\section{B. Impedance loss for large $\boldsymbol{g} / \boldsymbol{r}_{C}$}

Once bipolar flow is established in the SMP diode, the ions partially neutralize the beam charge, enabling a stronger magnetic pinch on axis [1]. The electron beam heats the plasma locally, drawing more current from the central (on-axis) portion of the plasma and distorting the contour of the plasma front. The shape of the plasma front is thus dependent on the angle of the beam pinch. This effect is seen in the density contours in Figs. 5(c) and 5(d) for the smallest aspect ratio, $g / r_{C}=1.12$. Ions are emitted perpendicular to the plasma front, following the potential gradient, and may have a significant transverse momentum when the plasma front angle is large with respect to the

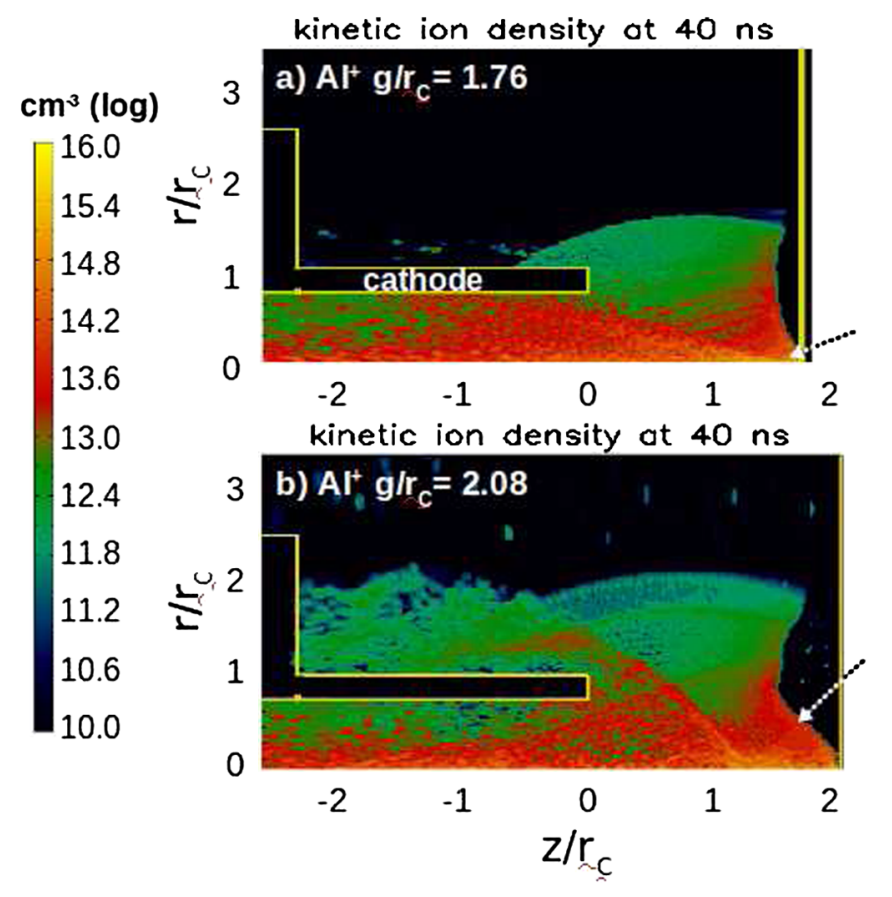

FIG. 7. Snapshots at $40 \mathrm{~ns}$ of kinetic ( $\geq 200 \mathrm{keV}$ ) ion density for the SMP diode with an aspect ratio of (a) $g / r C=1.76$ and (b) $g / r C=2.08$. Ions are drawn from an $\mathrm{Al}^{+}$anode plasma which fills the space between the ions and the anode. Arrows indicate the approximate trajectory of ions drawn from the plasma. The density contours are plotted on a $\log _{10}$ scale from $10^{10}-10^{16} \mathrm{~cm}^{-3}$. 


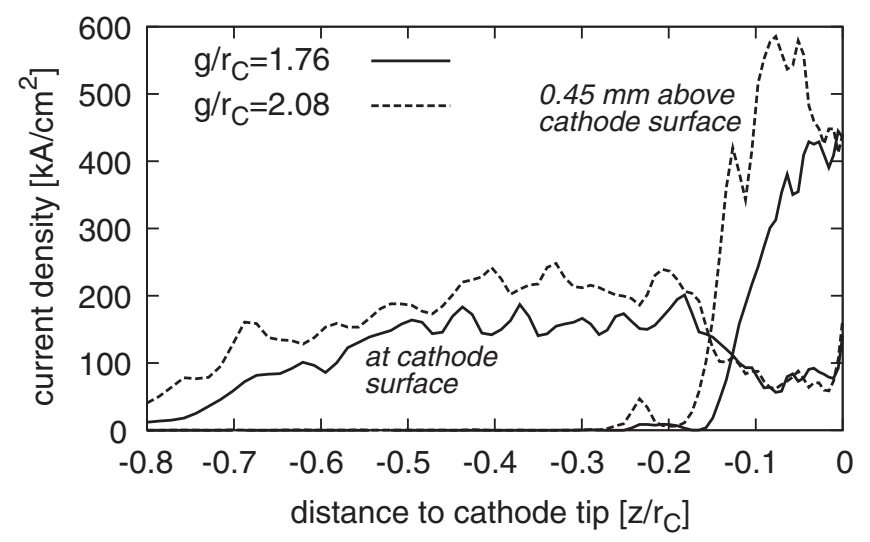

FIG. 8. Axial lineouts of the electron current densities along the cathode cylinder for $g / r_{C}=1.76$ (solid) and 2.08 (dashed) at $40 \mathrm{~ns}$ into the driving pulse. Two lineouts are taken for each geometry (one adjacent to the cathode surface and one $0.45 \mathrm{~mm}$ above) which show the current to be less tightly bound near the cathode tip. Both simulations are of an $\mathrm{Al}^{+}$anode plasma.

anode. High density contours of kinetic ions emitted from the plasma front near the axis can be seen in Figs. 5(a) and 5(b). The particles thus emitted in these figures are constrained to the interior of the diode due to the small aspect ratio. If their ratio of transverse-to-axial momentum exceeds $r_{C} / g$, ions may reach the outer surface of the cathode cylinder where their density may become non-negligible. This occurs in simulations of the $\mathrm{Al}^{+}$anode plasma for $g / r_{C} \geq 2$, where the larger aspect ratios contribute to both the larger plasma front angles and the reduced transverse momentum requirement.

An example of increased ion density outside the cathode cylinder is seen in the density contour in Fig. 7(b). This example is taken from the $g / r_{C}=2.08$ configuration at $40 \mathrm{~ns}$, after the impedance has begun to fall. The approximate trajectory of ions drawn from the plasma is indicated by an arrow. The ion density outside the cathode in this figure reaches levels of $3 \times 10^{13} \mathrm{~cm}^{-3}$. In contrast, the kinetic ion density contours for $g / r_{C}=1.76$ at $40 \mathrm{~ns}$, shown in Fig. 7(a), show a negligible ion density surrounding most of the cathode cylinder.

An increase in ion space-charge around the cathode draws more electrons from the length of the cathode cylinder. This is shown in the current density recorded along the cathode in Fig. 8, which is larger for $g / r_{C}=2.08$ than $g / r_{C}=1.76$. This increase in electron current leads to the fall in diode impedance shown in Fig. 4(b) for the larger aspect ratio. Like the gap-closure effect described in Sec. III A, this impedance-loss mechanism is not dependent on ion species in particular but on the combination of the operating parameters.

\section{CONCLUSIONS}

Experiments have shown that the SMP diode impedance decreases during the driving voltage pulse, with the most dramatic changes occurring at high and low values of $g / r_{C}$. Using a hybrid, implicit PIC technique to model anode plasma formation and evolution, two mechanisms are seen which can explain these falling impedances. The first mechanism is the expansion of the anode plasma into the diode A-K gap. The second is increased ion space-charge along the cathode.

In the first mechanism, the plasma formed on the surface of the anode expands into the diode A-K gap carrying the anode potential. The A-K gap width becomes time dependent, defined by the moving potential maximum. The diode impedance, which is proportional to the gap width, decreases in time. Simulations using different ion species have shown a predictable thermal velocity mass dependence to the expansion rate. This mechanism dominates for smaller A-K gaps.

In the second mechanism, ions with transverse-to-axial momentum in excess of $r_{C} / g$ may escape the region of dense electron space charge and form a non-negligible ion density outside the cathode. The increase in ion space charge along the cathode increases the electron current and decreases the diode impedance.

The application of the hybrid, implicit PIC technique has qualitatively reproduced the impedance behavior of the SMP diode observed in experiment. Future model improvements will include the effect of cathode plasma evolution and will determine the role of 3D instabilities. More detailed measurements of the ion species present, their ionization states, relative mixtures, and spatial extents would aid in confirming whether the two mechanisms presented here are responsible for the measured timedependent impedances.

\section{ACKNOWLEDGMENTS}

This work is supported by Sandia National Laboratories and the U.S. Department of Energy under Contract No. DOA-8910. Sandia National Laboratories is a multiprogram laboratory operated by Sandia Corporation, a wholly owned subsidiary of Lockheed Martin Corporation, for the U.S. Department of Energy's National Nuclear Security Administration under Contract No. DE-AC04-94AL85000.

[1] J. Threadgold, I. Crotch, D. Rose, and J. Maenchen, in Proceedings of the 14th IEEE International Pulsed Power Conference (IEEE, New York, 2003), p. 995.

[2] S. Swanekamp, G. Cooperstein, J. Schumer, D. Mosher, F. Young, P.F. Ottinger, and R. Commisso, IEEE Trans. Plasma Sci. 32, 2004 (2004).

[3] D. Hinshelwood, G. Cooperstein, D. Mosher, D. Ponce, S. Strasburg, S. Swanekamp, S. Stephanakis, B. Weber, F. Young, A. Critchley, I. Crotch, and J. Threadgold, IEEE Trans. Plasma Sci. 33, 696 (2005). 
[4] K. Hahn, N. Bruner, S. Cordova, R. Gignac, M. Johnston, J. Lechbee, I. Molina, B. Oliver, S. Portillo, J. Threadgold, T. Webb, D. Welch, and D. Ziska, IEEE Trans. Plasma Sci. 38, 2652 (2010).

[5] C. Litwin and R. Rosner, Phys. Rev. E 58, 1163 (1998).

[6] B. Oliver, K. Hahn, M. Johnston, and S. Portillo, Acta Phys. Pol. A 115, 1044 (2009).

[7] D. Hinshelwood, R. Allen, R. Commisso, G. Cooperstein, B. Huhman, D. Mosher, D. Murphy, P. F. Ottinger, J. Schumer, S. Swanekamp, S. Stephanakis, B. Weber, F. Young, I. Crotch, J. O'Malley, and J. Threadgold, IEEE Trans. Plasma Sci. 35, 565 (2007).

[8] S. Portillo, B. Oliver, S. Cordova, N. Bruner, D. Ziska, and D. Rovang, Sandia National Laboratories Technical Report No. SAND2007-6324, 2007.

[9] J. Maenchen, G. Cooperstein, J. O'Malley, and I. Smith, Proc. IEEE 92, 1021 (2004).

[10] D. Johnson, V. Bailey, R. Altes, P. Corcoran, I. Smith, S. Cordova, K. Hahn, J. Maenchen, I. Molina, S. Portillo, E. Puetz, M. Sceiford, D. V. de Valde, D. Rose, B. Oliver, D. Welch, and D. Droemer, in Proceedings of the 15th IEEE International Pulsed Power Conference (IEEE, New York, 2005), pp. 314-317.

[11] D. R. Welch, D. V. Rose, N. Bruner, R. E. Clark, B. V. Oliver, K. D. Hahn, and M. D. Johnston, Phys. Plasmas 16, 123102 (2009).
[12] D. R. Welch, D. V. Rose, M.E. Cuneo, R. B. Campbell, and T. A. Mehlhorn, Phys. Plasmas 13, 063105 (2006), LSP is a software product of ATK Mission Research.

[13] I. Langmuir and K. Blodgett, Phys. Rev. 22, 347 (1923).

[14] A. Critchley, A. Heathcote, and M. Johnston, IEEE Trans. Plasma Sci. 36, 1214 (2008).

[15] T. E. Madey, J. John, and T. Yates, J. Vac. Sci. Technol. 8, 525 (1971).

[16] S.W. Bellard and E. M. Williams, Surf. Sci. 80, 450 (1979).

[17] D. R. Welch and T. P. Hughes, Laser Part. Beams 16, 285 (1998).

[18] P. Menge and M. Cuneo, IEEE Trans. Plasma Sci. 25, 252 (1997).

[19] P. Redhead, The Physical Basis for Ultrahigh Vacuum (Chapman and Hall, London, 1968).

[20] H. Davis, R. Olson, and D. Moir, Phys. Plasmas 10, 3351 (2003).

[21] A. C. L. Fontaine, J. Phys. D 40, 1712 (2007).

[22] C. Vermare, J. Donohue, J. Labrouche, P. L. T. de Gabory, and D. Villate, IEEE Trans. Plasma Sci. 27, 277 (1999).

[23] D. Rovang, N. Bruner, M. Johnston, E. Madrid, J. Maenchen, B. Oliver, S. Portillo, and D. Welch, Phys. Plasmas 15, 093105 (2008). 